\title{
San Miguel de los Baños, un paraíso para rescatar
}

DOI: $\underline{\text { https://doi.org/10.33262/ap.v3i2.45 }}$

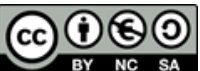

San Miguel de los Baños, a paradise to rescue

Lic. Aurelio Prieto Lee ${ }^{1}$, Ms.C. Massiel Martínez Carballo ${ }^{2}$ \& Dra. Maité Echarri Chávez ${ }^{3}$

\begin{abstract}
.
San Miguel de los Banos was known as "El Paraiso de Cuba" during the prerevolutionary stage (1900-1959) where the healing properties of its medicinal mineral waters were potentiated. Located in the municipality of Jovellanos, Matanzas, its underground waters favored local economic development between the years 1920 and 1929, with the construction of three hotels and a spa. Currently, San Miguel de los Banos offers great potential for the development of various tourist modalities. The objective of this research was to evaluate the current tourist-recreational potentialities of the town that contribute to the rescue of its values. The results of the study revealed that the territory has sufficient potential for the short-term implementation of health, nature and cultural tourism, which would bring great benefits.
\end{abstract}

Keywords: health tourism; local development; tourist towns; balneotherapy

\section{Resumen.}

San Miguel de los Baños fue conocido como "El Paraíso de Cuba" durante la etapa pre-revolucionaria (1900-1959) en donde se potencializan las propiedades curativas de sus aguas mineromedicinales. Ubicado en el municipio de Jovellanos, Matanzas, sus aguas subterráneas favorecieron el desarrollo económico local entre los años 1920 y 1929, con la construcción de tres hoteles y un balneario. Actualmente, San Miguel de los Baños ofrece

1 Universidad de La Habana, Facultad de Turismo. La Habana, Cuba. leesjun79@gmail.com 2 Universidad de La Habana, Facultad de Turismo. La Habana, Cuba. massielmc01@ gmail.com

3 Universidad de La Habana, Facultad de Turismo. La Habana, Cuba. echarrichavez@gmail.com 
grandes potencialidades para el desarrollo de varias modalidades turísticas. La presente investigación tuvo como objetivo evaluar las potencialidades turístico-recreativas actuales de la localidad que tributen al rescate de sus valores. Los resultados del estudio revelaron que el territorio cuenta con el potencial suficiente para la implementación a corto plazo del turismo de salud, naturaleza y cultural, lo que traería aparejado grandes beneficios.

Palabras claves: turismo de salud; desarrollo local; pueblos turísticos; balneoterapia.

\section{Introducción}

El mundo está cambiando, y eso se deja notar en muchos sectores. Uno de los más representativos de estos cambios es el turismo (Jiménez, 2015), que ya no es percibido como un privilegio ni como un lujo, sino como un derecho básico para recuperar el bienestar mental y físico, restableciendo la capacidad de llevar una vida activa (Solís, 2006).

El turismo de salud es para todas aquellas personas que busquen no sólo mantener sino incrementar su estado de salud durante sus vacaciones ya que la salud, sin lugar a dudas, es el bien más preciado de los turistas en vacaciones (Solís, 2006). En los últimos años, propiciado por los cambios sociales y las situaciones de estrés a las que se encuentra sometida la sociedad actual se ha ampliado la oferta de balnearios (Auren, 2013).

De acuerdo con lo planteado por Lorenzo \& Morales (2014) el desarrollo local es la interacción o relación entre agentes, sectores y fuerzas que interactúan en el territorio. Definiéndolo como un complejo proceso de concertación entre agentes, sectores y fuerzas que interactúan dentro de los límites de un territorio determinado (local, regional) con el propósito de impulsar un proyecto común que combine la generación de crecimiento económico, justicia social, sustentabilidad ecológica, enfoque de género, calidad y equilibrio espacial y territorial, cambio social y cultural, con el fin de elevar el nivel de vida y el bienestar de cada familia y ciudadano que viven en ese territorio o localidad.

El turismo se ha sido reconocido como un motor impulsor del desarrollo local, así lo fue en la primera mitad del siglo XX en la comunidad de San Miguel de los Baños, sin embargo, el paso de los años, unido a malas prácticas en la comunidad han propiciado un declive total de la actividad turística en la zona, trayendo aparejado un descenso en la calidad de vida de sus residentes. Por tanto, resulta necesario, un estudio que evidencie si San Miguel de los Baños posee valores históricos, económicos y socio-culturales que permitan un renacer del turismo.

San Miguel de los Baños, es un Consejo Popular perteneciente al municipio de Jovellanos, se encuentra geográficamente en el centro de la provincia de Matanzas, una de las más destacadas en el sector turístico de Cuba por su localización y su afamada playa de Varadero, 
donde se desarrolla a plenitud el turismo de sol y playa, siendo sin dudas el principal destino de la provincia.

La ya mencionada comunidad, se sitúa en la porción este de la región físico-geográfica de las Alturas del Bloque Bejucal-Madruga-Coliseo. Ocupa la parte alta de la cuenca del río San José, de la que constituye una subcuenca, con una extensión que alcanza 2965.28 ha y presenta características geográficas peculiares que lo destacan en el entorno provincial.

Los famosos manantiales brotan de una falla abierta entre las serpentinas, roca básica de esta zona, y la caliza. Las características geográficas de San Miguel de los Baños influyen grandemente en sus condiciones climáticas, sus vientos, en sus suelos, y sus aguas, las cuales conforman un ambiente tónico encantador, de bienestar y placidez, que, unido a su privilegiada posición en el centro de la provincia de Matanzas, convirtió desde siempre al lugar en un sitio de interés tanto de cubanos como de extranjeros, reconocido como "El Paraíso de Cuba" (Hernández, 2015).

\section{Metodología}

La presente investigación se considera cualitativa, sin embargo, se apoya en algunos elementos cuantitativos. Se han aplicado los principales métodos del nivel teórico y varias herramientas y técnicas cualitativas del nivel empírico, como parte de un estudio descriptivo y proyectivo, el cual pretende contribuir al rescate de San Miguel de los Baños como pueblo de interés turístico, a partir de la caracterización de la localidad, la identificación de sus potencialidades para dicha actividad y el establecimiento de lineamientos generales para un diseño futuro de estrategias y acciones con el ya mencionado fin. Basado en tres etapas fundamentales: preparatoria, de campo y final.

En la etapa inicial de la investigación se aplicó como método empírico el análisisbibliográfico que permitió identificar y consultar las fuentes de información bibliográfica necesarias, así como en extraer y recopilar los datos esenciales para la conformación de la investigación.

Se aplicaron como métodos teóricos:

Analítico-Sintético: Permitió obtener una visión global de las temáticas y categorías involucradas, evidenciando la relación existente entre ellas y descomponiendo en partes más sencillas el complejo todo que las integraba. Es decir, hizo posible llevar a cabo un análisis desde lo general hasta lo particular, apreciando las características de cada elemento y el resultado de su combinación. Además, hizo posible la comprensión de los principales factores que han intervenido en la formación de la identidad cultural de los sanmigueleños y el devenir histórico que ha tenido el poblado, descubriendo las relaciones entre estos y sus impactos sobre la realidad local. 
Histórico-Lógico: Accedió a identificar los antecedentes de la actividad balneológica en Cuba y, especialmente, en San Miguel de los Baños, estudiando su evolución y desarrollo, así como la de otras terminologías relacionadas al tema en cuestión, explicando la historia de su progreso o comprender el proceso de formación de la identidad de los sanmigueleños, facilitando las herramientas para la conformación de lineamientos que la rescaten como pueblo de interés turístico y le ofrezcan un valor añadido.

\section{Etapa de campo:}

Cuando se tenía conformado en marco conceptual y parte de la caracterización del objeto de estudio se aplicaron un conjunto de métodos empíricos que permitieron la obtención de resultados concretos para la realización de la investigación. Los métodos empíricos empleados fueron:

Observación directa no participante: El uso de este método permitió tener una percepción objetiva de la realidad del pueblo, realizando 2 visitas que posibilitaron reconocer la infraestructura, las actividades fundamentales que se desarrollan y el estado actual de los atractivos presentes en la localidad

En la presente investigación se seleccionó la variante de observación no participante, en la cual el investigador queda al margen de la situación a observar, captando todo lo potencialmente relevante sin la intervención ni manipulación del observador, permitiendo, de este modo, que los acontecimientos se desarrollen espontáneamente.

Entrevistas: Técnica aplicada a diferentes personas, de forma individual, algunos seleccionados intencionalmente y otros a los cuales las circunstancias en las que se desenvolvió la investigación hicieron posible consultar. De forma general, fueron entrevistados algunos actores clave dentro del desarrollo de la localidad, pobladores, trabajadores de la esfera turística y de la salud, así como profesionales con conocimientos generales y específicos de la comunidad.

Consulta a especialistas: Se consultó a diferentes especialistas respecto a los principales temas relacionados con la investigación, cuyos criterios fueron clave para la identificación de diferentes aspectos que tienen incidencia sobre el desarrollo del territorio, ya sea de forma positiva o negativa. Se llevó a cabo en forma de entrevista semi-estructurada y para su selección de los especialistas se tuvo criterios como la vinculación a la actividad médica ligada a tratamientos de aguas mineromedicinales y otras terapias relacionadas, ocupar cargos de dirección en instituciones gubernamentales, culturales o recreativas del territorio sanmigueleño

Inventario de Recursos: Los recursos fueron inventariados utilizando el modelo del Manual para la Formulación del Inventario de Recursos Turísticos a Nivel Nacional (Fase I - 
Categorización) del Ministerio de Comercio Exterior y Turismo del Perú (MINCETUR), en el que se identifican cada recurso en una ficha, especificándose en la misma el número, nombre del recurso turístico, ubicación, categoría, tipo, subtipo, descripción, particularidades, estado actual, tipo de visitante, acceso hacia el recurso, tipo de ingreso, época propicia de visita al recurso, infraestructura dentro del recurso y actividades desarrolladas dentro del recurso. Este inventario recoge fundamentalmente los bienes histórico-culturales y naturales que atesora la localidad.

Evaluación turística: Se llevó a cabo una evaluación turística del área de tipo funcional, higiénico-sanitaria, técnico-económica, conservacionista y estética, partiendo de conceptos previamente establecidos. Las valoraciones se realizaron con una escala ascendente de 1 a 5, siendo 1 la puntuación más baja y 5 la más alta. El promedio de las evaluaciones permitió determinar los recursos que pueden ser integrados en la conformación de una oferta turísticorecreativa atractiva, novedosa y de interés al mercado objetivo.

\section{Etapa final:}

En esta etapa se hizo pertinente organizar y procesar toda la información obtenida de la aplicación de los métodos empíricos durante la etapa de campo.

Para el desarrollo de esta etapa de la investigación se ejecutaron como métodos teóricos:

Análisis-síntesis: A través de este método se analizó el objeto de estudio, San Miguel de los Baños, obteniendo información primaria necesaria para su propuesta como pueblo de interés turístico y sintetizando los resultados arrojados del análisis del inventario del objeto de estudio.

Histórico-lógico e Inductivo- deductivo: En esta etapa se concibe la investigación como un Proyecto de Desarrollo pues con el mismo, se obtiene la propuesta de un pueblo como interés turístico. A su vez, ésta ha sido concebida como un producto, destinado a rescatar los valores histórico-culturales y naturales existentes, ya que constituye una nueva oferta para llamar la atención de los visitantes, y ofrecería al destino nuevas oportunidades competitivas. El resultado de este trabajo es sólo la concepción de proponer el desarrollo turístico de San Miguel de los Baños, por lo que no se incluyen análisis de demanda, factibilidad o comercialización. El autor deja abierta la posibilidad de que se hagan, en estudios posteriores, los análisis necesarios para la implementación y puesta en práctica de la misma como oferta turística.

En todas las etapas de la investigación se aplicaron los siguientes métodos:

Inductivo-deductivo: permitió relacionar las referencias de autores, elementos teóricos y demás información, arrojando conclusiones que permitieron el desarrollo efectivo de la 
investigación y que la información se procesara de la forma más sencilla y clara para el lector.

Histórico-lógico: permitió el estudio y planteamiento cronológico de las citas de autores en los planteamientos teóricos y de la caracterización del poblado de San Miguel de los Baños, así como de la elaboración de los modelos de entrevistas y el inventario, logrando en todo momento un sentido más lógico y coherente en el desarrollo de la investigación.

\section{Resultados}

A pesar de su abandono, las aguas mineromedicinales de San Miguel de los Baños son únicas en su tipo en Cuba que, unido a sus tradiciones y la confluencia de cultura, historia y religión presentes en sus tierras, hacen de este lugar un centro de placidez, salud, descanso y recreo, con un elevado grado de autenticidad. Estos elementos hacen del sitio un terreno con gran potencial según las tendencias mundiales hacia el turismo ecológico, de salud, en bienestar y calidad de vida.

Rodeado de elevaciones, con un microclima propio y vegetación autóctona se convirtió en el pasado, en el principal de los balnearios del país. Aún conserva sus encantos naturales, pero los valores patrimoniales que identificaron su tradición balnearia, se encuentran deteriorados por falta de mantenimiento; es el caso del Balneario con estilo de renacimiento francés y otros hoteles que brindaban servicios de hospedaje a los turistas y visitantes, conjuntamente con muchos de los inmuebles y casas de huéspedes, cuya tipología constructiva distinguían la ciudad. Aunque no es posible tomar baños, como lo fue en el pasado, se puede acceder a una instalación en la parte trasera del antiguo Balneario, donde los visitantes y los pobladores del lugar llenan vasijas del milagroso líquido.

Tiene geográficamente una magnífica situación. Se puede decir que, en un recorrido de 140 km hasta el mismo, se conduce a La Habana, quedando tan sólo a $40 \mathrm{~km}$ de la Ciudad de Matanzas y a 30 de Cárdenas. Se encontraba en el camino obligado de la famosa playa de Varadero, lo que casi llevaba al excursionista a realizar una parada en dicho Balneario, para descansar y tomar un refrigerio.

Todo lo anterior, sumado a los recursos histórico-culturales y naturales que posee, la tranquilidad y pasividad de sus pobladores, integrados en su mayoría por trabajadores de la artesanía con un profundo sentido de pertenencia hacia su localidad y las oportunidades de desarrollar acciones encaminadas a incorporar servicios y una infraestructura con fines turísticos, lo convierten en un excelente destino para el impulso de actividades turísticorecreativas, las cuales pueden desplegarse como ofertas complementarias a un turismo de salud.

Sin embargo, a pesar de sus ya mencionados valores y los importantes esfuerzos de algunos pobladores por revitalizar el pueblo para su desarrollo turístico, rescatando y poniendo en vigor esos bienes históricos-culturales y naturales que atesora, no ha recibido el apoyo de las 
entidades pertinentes, quienes se justifican, fundamentalmente, con una falta de presupuesto y cercanía a unidades militares, lo que dificulta la restauración y el mantenimiento de los atractivos más valiosos, los cuales exhiben un elevado grado de desgaste.

Aunque a raíz del descubrimiento de las aguas mineromedicinales se estableció una importante infraestructura para la actividad de servicio de alojamiento, con una buena cantidad de casas de descanso para vacacionar, y se desarrolló el turismo en el territorio, la situación actual muestra un escenario diferente.

De los cuatro hoteles que San Miguel contaba en sus inicios, hoy solamente se mantiene funcionando el Hotel San Miguel, brindando exclusivamente servicios gastronómicos, mientras que el Gran Hotel Balneario, joya arquitectónica del poblado, se encuentra en estado ruinoso, necesitando de una rehabilitación capital urgente. Se han presentado varios proyectos de reparación por parte de instituciones estatales, pero ninguno ha logrado una mejora visible. Los pocos turistas que visitan el pueblo, van atraídos por la historia que encierra el Balneario y por las propiedades de las aguas de sus manantiales. Acuden también personas de toda el área cercana y hasta de lugares distantes del país.

La industria extrahotelera, está caracterizada por un punto de venta de gastronomía estatal en el Hotel San Miguel y 3 cafeterías particulares que ofrecen comida rápida. No obstante, vale resaltar que estas últimas presentan variedad de productos, destacándose en este sentido, "La Guarina", mientras que la situada en los bajos del hotel, brinda solamente servicio de desayuno y venta de alimentos elaborados en el horario del almuerzo.

En resumen, la actividad turística en el territorio es casi nula, causada no sólo por las dificultades para el alojamiento y los servicios de restauración, sino por la ineficaz gestión de los recursos histórico-culturales, naturales y arquitectónicos de la comunidad, en manos de los organismos administrativos municipales y provinciales. En cuanto a la transportación local, es bastante estable caracterizada por vehículos particulares como camionetas y taxis; pero si se alcanza un desarrollo turístico, se deben crear nuevos servicios en aras de facilitar los flujos de turistas hacia el pueblo.

San Miguel de los Baños, posee grandes potencialidades para el desarrollo de un turismo de salud, pues posee recursos naturales importantes que lo complementan, en especial su atractivo de las aguas mineromedicinales. Su desarrollo podría satisfacer las necesidades de los que por razones de salud requieren de un tratamiento clínico- terapéutico en un lugar tan apropiado como este que posee propiedades curativas por su clima y la calidad de sus aguas. La acción de los baños resulta de gran beneficio por sus efectos terapéuticos, reconocidos por la ciencia como verdadero tónico sobre el sistema nervioso, la circulación, el hígado, los riñones y la respiración; excelentes para las vías digestivas y urinarias, útiles para combatir la anemia, diabetes, gastralgia, dispepsia, urticaria, clorosis, nefritis, neurastenia y otras enfermedades. 
Este espacio turístico cuenta también con importantes recursos que le permiten desarrollar el turismo histórico-cultural, ejemplo de ello es el fuerte movimiento artesanal, las arraigadas costumbres religiosas donde en cada Semana Santa se realizan peregrinaciones hasta el Cristo de Jacán, acto que demuestra la identidad de sus pobladores. También constituye un erario en la arquitectura antigua y de madera. Constituyó un escenario de la realización de nuestras guerras por la independencia y posee disímiles cuevas y lugares que aún conservan su valor histórico y nos brindan informaciones arqueológicas sobre nuestros antepasados.

La localidad dispone de todos los elementos para poner en práctica el ecoturismo. Cuenta con bellísimos paisajes naturales, rodeados de una vegetación tropical con un bajo impacto ambiental por el ser humano, lugares de observación de una exclusiva flora y fauna, áreas naturales de elevados valores estéticos-paisajísticos, yacimientos de aguas mineromedicinales, ríos y arroyos, montañas hermosas; además del alto grado de conciencia alcanzada por la población relacionada con la conservación del medio ambiente natural, lo que ha contribuido a la posibilidad de desarrollar actividades turístico-recreativas vinculadas al mejor conocimiento de la naturaleza y de la cultura.

Por último, es un importante destino turístico para el desarrollo del turismo comunitario, ya que éste constituye una vía para generar ingresos a partir de las actividades económicas cotidianas, utilizando los recursos culturales, naturales y locales de la región. Además, permite a los habitantes convertirse en guías turísticos y prestar servicios a los visitantes que reciben, generando una participación más activa del turista dentro de los hábitos y costumbres de la localidad. De esta forma, se consigue reanimar el poblado a través del turismo, aumentar y garantizar la permanencia de formas de vida tradicionales, contribuye a la generación de empleo, propicia el intercambio sociocultural, se pone en valor el patrimonio natural y cultural disponible en la comunidad y, al mismo tiempo, se crea conciencia y respeto al ambiente cultural y comunitario.

Con los resultados del inventario de recursos, se pudo apreciar las potencialidades del territorio para el desarrollo del turismo, acompañado de las manifestaciones históricas culturales y el folclore de la localidad, pues no son pocos los elementos de valor que atesora la misma, los cuales no han sido aprovechados para el despliegue de actividades turísticorecreativas del territorio.

Se logró evaluar la carencia de una infraestructura turística, técnica y de equipamiento que le permita hacer frente a corrientes turísticas, aunque cabe señalar que en la localidad es posible la organización de excursiones opcionales que formen parte de paquetes de otros destinos. La extrahotelera es ínfima, donde las instalaciones y servicios de estas unidades no responden plenamente a los requerimientos necesarios para enfrentar una demanda del mercado turístico convencional.

El pueblo cuenta con su propia red de alcantarillado, de ahí que las condiciones higiénicosanitarias fueran valoradas de buenas. El territorio y sus alrededores poseen una buena higiene, los desechos sólidos son recogidos a tiempo y sus calles siempre lucen limpias. El 
sector de los manantiales del Balneario no está contaminado, lo que permite el uso medicinal de las mismas.

De manera general, se puede plantear que el pueblo, a pesar de estar bendecido por la naturaleza y el patrimonio histórico-cultural, revela altos grados de deterioro en sus construcciones más valiosas, producto de no ser gestionado adecuadamente. No obstante, no tiene alteraciones o rupturas del tejido histórico urbano. Es precisamente en este elemento donde radican las potencialidades de esta localidad, es decir, en la permanente relación entre el fenómeno constructivo y urbano con una comunidad de cultura tradicional. La conservación en la actualidad de la estructura urbanizada proveniente del pasado y el reconocimiento de su identidad cultural es de vital importancia para su análisis desde un punto de vista turístico para su rescate y puesta en valor.

En la Tabla 1 se muestran los resultados de la evaluación turística realizada a siete sitios que se consideran de obligada visita en el territorio sanmigueleño (Gran Hotel Balneario, Sector de Manantiales, Iglesia de San Miguel, Ermita del Cristo de Jacán, Loma de Jacán, Casa de la Cultura y el Núcleo Fundacional). Para ello se han seguido los criterios de tipo funcional, higiénico-sanitario, técnico-económico, conservacionista y estético sugeridos por Salinas (2003). Se asignaron valores entre 1 y 5 puntos de manera que el máximo posible a alcanzar fuera de 35 puntos. El análisis de los resultados se realizó a partir de sumar los promedios de las evaluaciones individuales en cada uno de los aspectos, lo que tributaría a una evaluación general del pueblo. Esta valoración fue posible mediante la suma de los resultados finales de cada recurso, siendo de 175 puntos la máxima puntuación posible a alcanzar.

Tabla 1: Evaluación turística

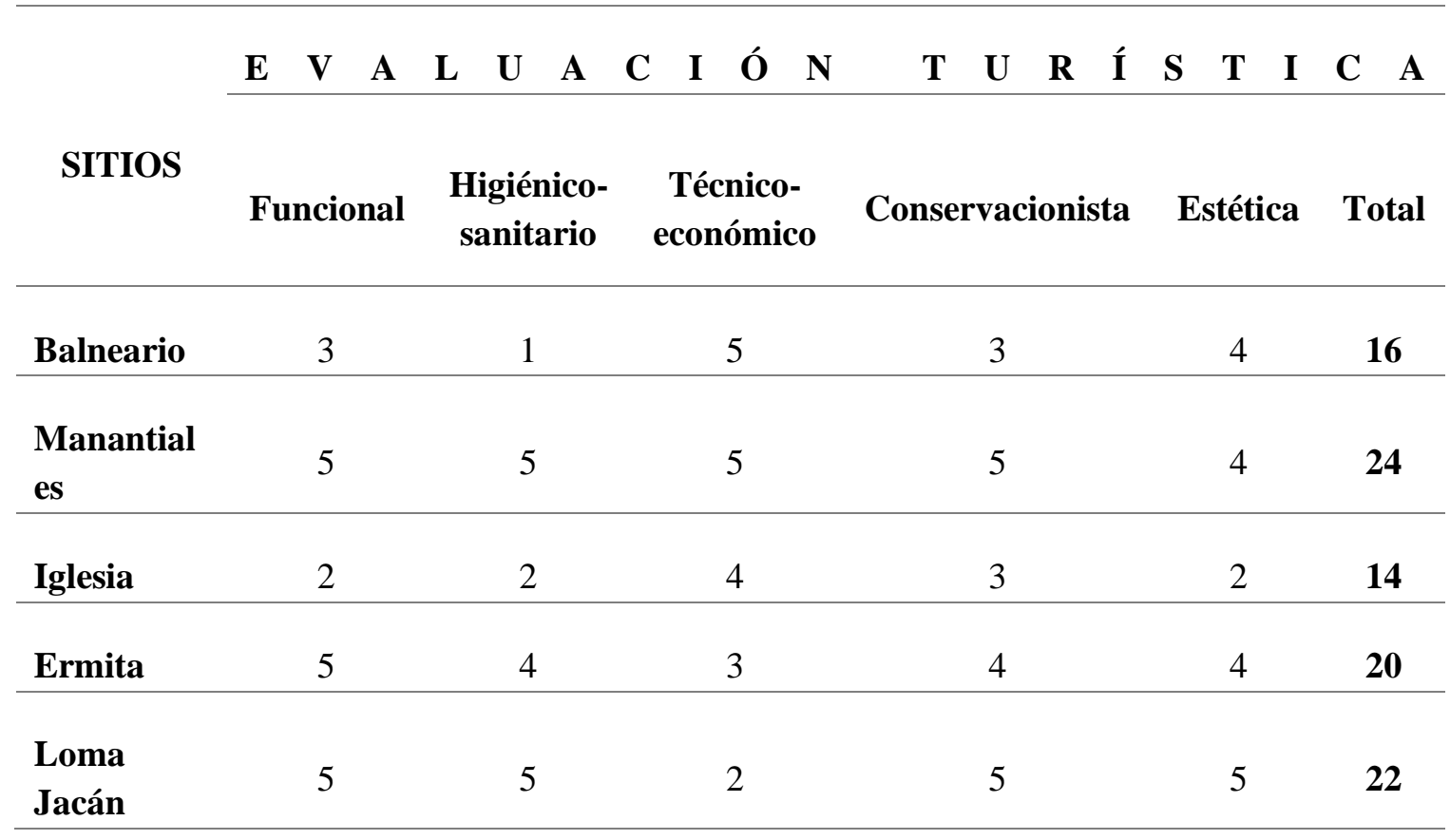




\begin{tabular}{lcccccc}
$\begin{array}{l}\text { Casa de la } \\
\text { Cultura }\end{array}$ & 5 & 5 & 3 & 5 & 4 & $\mathbf{2 3}$ \\
\hline $\begin{array}{l}\text { Núcleo } \\
\text { Fundacion } \\
\text { al }\end{array}$ & 5 & 5 & 5 & 5 & 4 & $\mathbf{2 4}$ \\
\hline Total & 30 & 29 & 27 & 30 & 27 & $\mathbf{1 4 3}$ \\
\hline
\end{tabular}

Como se puede apreciar, la máxima evaluación fue alcanzada por los Manantiales y el Núcleo Fundacional, ambos con 24 puntos, siendo la Iglesia de San Miguel el peor evaluado, con solo 14 puntos.

Los aspectos funcional y conservacionista obtuvieron puntuaciones favorables, 30 puntos cada uno, lo que representa un aproximado de $86 \%$ de la máxima puntuación a alcanzar. Esto demuestra de una forma u otra, que cada uno de los sitios seleccionados para la evaluación cumple con su objeto social, logrando varios de ellos, la exclusividad y una inminente necesidad de conservación.

El indicador higiénico-sanitario alcanzó 29 puntos, para un 83\% de acumulado; mientras que las cifras más bajas están asociadas a los factores técnico-económico y estético con 27 puntos cada uno, lo que representa un $77 \%$, ocasionado fundamentalmente por la escasez de recursos monetarios para llevar a cabo el financiamiento de estas obras.

En cuanto a la estética, el pueblo está en un estado de opaques total, a pesar de la existencia de valores patrimoniales y elementos atrayentes.

Seguidamente, la evaluación general del pueblo de San Miguel de los Baños resultó de 143 puntos, lo que se corresponde con un $81,7 \%$ de la puntuación total, resultado que demuestra que aun cuando es necesario invertir en determinados aspectos que se han explicado a lo largo de la investigación, como son las instalaciones de alojamiento y restauración, la rehabilitación total del Balneario, así como en otros atractivos que posee la zona, que pudieran incorporarse a una nueva evaluación, con una adecuada gestión, se puede aprovechar su alto potencial para el desarrollo de actividades turístico-recreativas.

Un análisis comparativo entre los principales recursos de interés turístico, nos muestra los resultados siguientes:

Los principales recursos turísticos de San Miguel de los Baños son, en primer lugar, sus aguas mineromedicinales y el núcleo fundacional (ambos con 24 puntos), debido a que en la provincia de Matanzas no hay otro destino turístico que tenga la misma topología de oferta y es motivo de atracción potencial de turistas al municipio. Por otro lado, existen en la localidad, las casas de madera que, con un estilo neoclásico, poseen una confluencia de culturas foráneas y representan la historia del arte constructivo en Cuba. Se suma también la 
Casa de la Cultura (23 puntos), con una historia y trayectoria muy interesante, ejemplo de ello fue la creación del Taller Literario Jacán, con significativos logros. El estado funcional, higiénico-sanitario y conservacionista son las principales condicionantes para sus resultados.

Dentro de los recursos turísticos naturales, se destaca la Loma de Jacán (22 puntos), dando un ambiente agradable a sus visitantes con una determinada flora y fauna, dando lugar a paisajes pintorescos.

La Ermita del Cristo de Jacán, por su parte, tiene una elevada representatividad religiosa, ya que son muchos los visitantes que allí acuden por el valor que presenta, relacionado con la historia de San Miguel. Se destaca en este recurso su estado funcional para la cual fue diseñada.

La Iglesia, con 14 puntos fue la de peor evaluación, junto con el Balneario, con 16 puntos. En este último, influyeron en sus resultados, las condiciones higiénico-sanitarias en el que se encuentra, que no son las mejores; en el caso del objeto social para el que fue creado y su estado conservacionista, no mostraron resultados favorables, debido a su mal estado de conservación, por lo que se requiere de una fuerte inversión para su recuperación. Sin embargo, constituye un atractivo el hecho de que el balneario sea una réplica del "Casino de Montecarlo", siendo arquitectónicamente único de su tipo en el país.

En el caso de la Iglesia de San Miguel, sus resultados se deben también a las malas condiciones en que se encuentra, declarada actualmente por las autoridades locales en peligro de derrumbe. Hoy, está sometida a un proceso de rehabilitación capital. No obstante, es innegable el valor arquitectónico, histórico y religioso de la misma. San Miguel de los Baños tiene potencial para ser un sitio turístico más que atractivo, y tanto la Iglesia como la escalinata del Cristo serían pilares fundamentales para que este sueño se materialice.

A continuación, se enumeran las principales Fortalezas, Debilidades, Oportunidades y Amenazas que fueron detectadas, después de haber hecho un profundo análisis de sus características internas y un estudio de la situación externa, en trabajo conjunto con los especialistas consultados, y posteriormente se muestra la matriz de impactos cruzados.

\section{Análisis Interno:}

\section{Fortalezas}

F1- Exclusividad de las aguas mineromedicinales de San Miguel de los Baños.

F2- Prestigio Nacional como antiguo Paraíso de Cuba.

F3- Idoneidad para el desarrollo del turismo de salud.

F4- Potencialidad para el desarrollo de diversas actividades turísticas-recreativas y modalidades.

F5- Potencialidad del recurso humano. 
F6- Autogestión del 1\% por parte del Consejo de Administración Municipal (CAM) para iniciativas de desarrollo local.

F7- Cercanía del poblado al principal destino turístico de sol y playa de Cuba (Varadero).

Debilidades

D1- Presupuesto insuficiente para el emprendimiento de obras constructivas en el Balneario de San Miguel y de la comunidad en general.

D2- Deterioro del espacio urbano y de las infraestructuras existentes.

D3- Conformismo y desmotivación de la población local.

D4- Descomercialización del poblado como destino turístico.

D5- Falta de interés por parte del gobierno local.

D6- Existencia de una sola vía de acceso al lugar.

D7- Insuficiente capacidad de alojamiento.

\section{Análisis Externo:}

\section{Oportunidades}

01- Perfeccionamiento de la gestión económica del territorio.

O2- Posibilidades de financiamiento nacional e internacional a través de proyectos.

O3- Escaso aprovechamiento de las modalidades no tradicionales en el país.

O4- Aumento de la preferencia por parte de la demanda hacia las modalidades de turismo de salud.

O5- Sistema político-social estable y alto grado de seguridad ciudadana y sanitaria.

O6- Tendencia internacional hacia el desarrollo del turismo comunitario.

Amenazas

A1- Oferta de salud comercializada a través de Servicios Médicos Cubanos.

A2- No inclusión de ofertas de balneario en las Agencias Receptivas.

A3- No inclusión del poblado en la Cartera de Oportunidades y Planes de Desarrollo.

A4- Nuevas medidas implantadas por la Administración Trump.

A5- Lento desarrollo del proceso inversionista.

A6- Existencia de competidores en la zona occidental del país (San Diego de Los Baños, Santa María del Rosario y Elguea). 
ISSN: 2773-7330 abril-junio, 2021

A7- Pandemia del Coronavirus detiene la actividad turística en el país e impacta negativamente en los viajes a nivel global.

Matriz de Impactos Cruzados

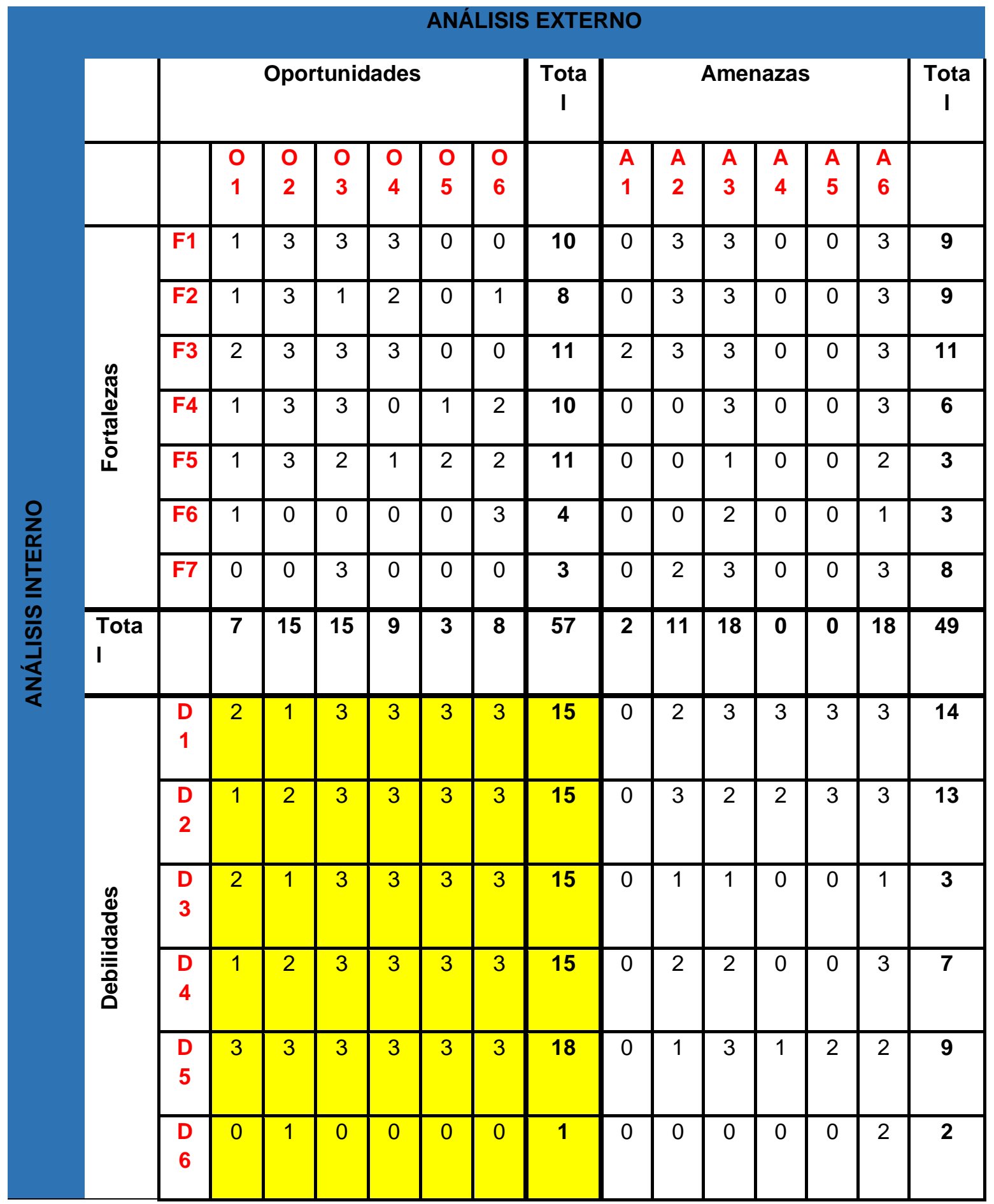




\begin{tabular}{|l|l|l|l|l|l|l|l|l|l|l|l|l|l|l|l|}
\hline & $\begin{array}{l}\mathrm{D} \\
7\end{array}$ & 1 & 2 & 1 & 3 & 0 & 3 & 10 & 0 & 0 & 2 & 0 & 3 & 2 & 7 \\
\hline $\begin{array}{l}\text { Tota } \\
\text { I }\end{array}$ & & 10 & 12 & 16 & 18 & 15 & 18 & 89 & 0 & $\mathbf{9}$ & $\mathbf{1 3}$ & $\mathbf{6}$ & $\mathbf{1 1}$ & $\mathbf{1 6}$ & $\mathbf{5 5}$ \\
\hline
\end{tabular}

San Miguel de los Baños presenta excelentes potencialidades para la puesta en marcha de actividades turístico-recreativas, sin embargo, la existencia de competidores en la zona occidental del país y la no inclusión del poblado en la Cartera de Oportunidades y Planes de Desarrollo, pudieran agredir significativamente su proceso de desarrollo local. Es preciso, superar las debilidades. La falta de interés por parte del gobierno local, unido al presupuesto insuficiente para el emprendimiento de obras constructivas en el Balneario y de la comunidad en general; imposibilita la visión integral del destino. Esto ha sido resultado del deterioro, tanto del espacio urbano, como de las infraestructuras existentes, del conformismo y la desmotivación de la población local y de la descomercialización del territorio como zona turística.

El pueblo debe potenciar a tope las fortalezas con que cuenta, es decir, ganar el máximo provecho al hecho de contar con la capacidad para desarrollar el turismo de salud, así como el turismo cultural y naturaleza, y la potencialidad de su recurso humano; apoyado de la exclusividad de sus aguas mineromedicinales dentro de la región.

Por otro lado, es importante aprovechar plenamente las oportunidades que brinda el entorno, como son las perspectivas de financiamiento nacional e internacional a través de proyectos y el insuficiente aprovechamiento de las modalidades no tradicionales en el país.

Es obligación de los organismos implicados (Poder Popular, Ministerio de Salud Pública y Ministerio de Cultura) cuya gestión hasta hoy ha sido nula, involucrarse en el asunto, para lograr un verdadero despliegue del turismo sostenible en la comunidad, con el fin de recobrar la rica cultura local que atesora San Miguel de los Baños, subsanando las deficiencias con la restauración del Balneario, y de otros atractivos de la localidad; la apertura de nuevos espacios para la recreación, así como la construcción de instalaciones para el hospedaje y el impulso a la actividad por cuenta propia que complemente esta actividad; lo que traería consigo no sólo beneficios económicos, sino también una mejora de la calidad de vida de los pobladores y la formación de una conciencia más sólida sobre la importancia de preservar los recursos naturales y patrimoniales en el territorio.

Estas recomendaciones deben ser tomadas en cuenta, con el objetivo de aprovechar las ventajas que ofrece la localidad, el interés del MINTUR en la creación de ofertas municipales para la diversificación del producto nacional o la tendencia creciente, sobre todo en personas de la tercera edad, por la combinación de los tratamientos de salud con elementos de la cultura e idiosincrasia locales, sumados a actividades de intercambio con la naturaleza. 
Las propuestas de mejora deben orientarse tanto a disminuir las debilidades, reforzar y mantener las fortalezas a la búsqueda de oportunidades convenientes para las capacidades del destino, como a proveer una defensa para atenuar las amenazas externas.

\section{Lineamientos generales para el diseño de una estrategia turístico-recreativa en el pueblo de San Miguel de los Baños.}

Los valores naturales que caracterizan la localidad de San Miguel de los Baños hacen de ésta, un área significativa que se destaca en el contexto provincial. Los elementos históricos y arquitectónicos son los que hacen más singular y atractivo a este pueblo, ya que fue catalogado en su momento "El Paraíso de Cuba" por su belleza, tranquilidad, magníficas construcciones y por sus aguas mineromedicinales. Éstas últimas eran y son conocidas en todo el país, incluyendo sus maravillosas instalaciones, destacándose su imponente Balneario. Son estos, elementos suficientes para plasmar una estrategia, que sea capaz de promover el lanzamiento de actividades turístico-recreativas en el territorio, favoreciendo su rescate como pueblo de interés turístico. Sin embargo, es necesario controlar y superar las debilidades existentes, que limitan o reducen la capacidad del desarrollo efectivo de la estrategia del poblado, para lograr el aprovechamiento óptimo de los recursos.

En este sentido, se han trazado líneas de acción, formando un equilibrio armónico con la naturaleza, con una propuesta integral protegiendo el entorno natural, que persigue los siguientes objetivos:

- Crear las infraestructuras que permitan restablecer las mínimas condiciones para que San Miguel de los Baños pueda operar, recuperarse y crecer a corto y mediano plazo.

- Mejorar el espacio natural, mediante la revitalización de la flora y fauna de la zona, incorporando valor agregado al área objeto de estudio.

- Reportar beneficios económicos que repercutan directamente sobre la población local, mejorando la calidad de vida de la misma y generando nuevas capacidades de empleo.

- Educar a los residentes y turistas acerca de la importancia de conservar los valores naturales, histórico-culturales y sociales del área, a través de programas de educación ambiental.

- Impulsar el desarrollo local, a través de un turismo sostenible.

Se pretende involucrar, de una forma u otra, todas las riquezas del territorio, para lograr la reanimación y rescate integral de San Miguel de los Baños, siendo los pobladores los verdaderos protagonistas de su desarrollo, obteniendo provecho de los recursos endógenos, sin causarles daños con su utilización ni provocar cambios negativos en sus labores principales (agropecuaria, forestal y artesanal). A continuación, se explican los lineamientos atendiendo al análisis DAFO, para dar solución a las problemáticas que hoy pone en peligro 
la integridad urbana del poblado, proyectando un futuro mejor propiciando la dinamización y cualificación para la gestión, desarrollo y sustentabilidad del turismo. Para ello se ha tenido en cuenta los antecedentes de propuestas para el desarrollo turístico del territorio. Entre ellos, sobresalen: "Propuesta de Plan de Manejo de San Miguel de los Baños", llevado a cabo por el Lic. Ciencias Ambientales Isaac Llinás, trabajo realizado por el CITMA, en conjunto con la Universidad de Matanzas Camilo Cienfuegos y la Universidad de Girona (2007); y "Rehabilitación de Centros Históricos en la Provincia de Matanzas, Ilustraciones para un Rescate Integral del Patrimonio", cuyo autor es el arquitecto Erik Denis Falcón, correspondiente al ISPJAE (2008).

\section{Recuperación integral del Gran Hotel Balneario de San Miguel de los Baños.}

Durante la evolución de este trabajo, se ha hecho mención a que actualmente el Balneario no brinda ni presta servicio alguno, debido a que se encuentra deshabilitado en toda su estructura. Rescatar esta reliquia arquitectónica, junto a sus manantiales, traería al poblado su verdadera esencia para el que fue diseñado.

El balneario como fuente de vida para el poblado desde sus inicios, sería por su fuerza renovadora, la primera etapa dentro de una estrategia a corto plazo, por su importancia dentro de este proyecto. No obstante, su recuperación es la fase de más coste, pero se piensa que si el turismo funciona sería fácil encontrar un inversionista que esté interesado en recuperarlo. Una forma de reutilizar este espacio pudiera ser la creación de un SPA, incorporando un centro de masajes y fisioterapia, dando así cabida a las nuevas inquietudes por la salud del pueblo cubano y del mundo entero, el cuál envejece cada vez más al aumentar la esperanza de vida. Esta acción se ejecutaría siempre respetando la integridad de su imagen, sumando nuevos valores al edificio, modernizando toda la estructura funcional, para integrar al Balneario, funciones modernas durante el período de tratamientos.

Se debe, además, incorporar una amplia gama de servicios con atención personalizada apoyado de profesionales en el tema, unido a espacios más acogedores, decoración innovadora y diversidad de colores. El Balneario ha de adoptar una nueva imagen, destinada a la curación y al bienestar de los clientes, alejado del ambiente frío de los hospitales tradicionales.

Se necesita de una inyección financiera inicial que permita recuperar la instalación para comenzar a brindar servicios e iniciar un autofinanciamiento para que sea capaz de cubrir sus gastos e ir incrementando la cantidad y calidad de los tratamientos bajo la condición de una mejora continua en busca de la excelencia.

\section{Propuestas de infraestructura de alojamiento:}

- Ampliar la oferta de casas de descanso de empresas estatales.

Esta propuesta se cree que es la oferta que se tiene que potenciar más, ya que representa un turismo nacional, que viene buscando descanso y constituirse un importante canal de 
promoción nacional. También supone que el mismo Gobierno invierta en San Miguel y reconozca sus potencialidades.

- Campismo.

Hace años el poblado contaba con un campismo con piscina natural de aguas mineromedicinales, actualmente se encuentra en un estado de abandono total, pero es viable su reapertura ya que es de fácil arreglo y de bajo costo. Constituye una buena forma de atraer turistas nacionales, ya que este modelo turístico es muy usado por la población cubana. Además, no es el típico campismo de playa, sino uno integrado en la naturaleza.

- Recuperar el Hotel San Miguel.

El hotel se encuentra en mal estado, pero no en ruinas y sería de fácil recuperación. Podría dar cabida, en un primer momento, a un pequeño flujo de turistas nacionales o internacionales.

- Adecuación de casas de agroturismo.

La contaminación que existe en las grandes ciudades y la vida de estrés que se rige en ellas hacen que cada vez más se busquen ofertas de turismo de tranquilidad e integración con la vida del campo y la naturaleza. La oferta de casas de agroturismo es un buen reclamo para satisfacer estas necesidades, haciendo las actividades que realiza un campesino durante su vida diaria.

Se cree que San Miguel tiene gran potencialidad en este sentido. Esta actividad está muy ligada con las rutas a caballo, ya que se pueden utilizar las instalaciones del centro de agroturismo (establo) para adecuar a los equinos y realizar los recorridos turísticos que se proponen más adelante.

Esta actividad es factible de llevar a cabo a corto o mediano plazo y sin grandes inversiones económicas y, además, dan singularidad a la oferta turística.

- Casas particulares.

Esta tipología de alojamiento se cree que es muy buena para el pueblo, ya que ayudará a la reconstrucción de las mismas casas que oferten este producto $\mathrm{y}$, de este modo, no requiere una gran inversión inicial. Pero en todo caso, se propone a mediano-largo plazo, ya que primero se tendrá que precisar si el desarrollo turístico funciona en San Miguel, el perfil del turista potencial que está interesado y un estudio económico para definir su viabilidad.

\section{Rehabilitación de las casas coloniales para el poblado de interés turístico.}

Resulta evidente cuán grande es el tesoro arquitectónico presente en el conjunto de casas de madera, por tanto, sería provechoso su recuperación, respetando el contexto tanto paisajístico como urbanístico. Este conjunto, que poca comparación tiene entre los más bellos ejemplos de viviendas de su tipo no sólo en Cuba, sino en todo el Caribe, se está poco a poco perdiendo y quedando opacado las bellas edificaciones de madera. 
Se rehabilitarán las casas de madera típicas del lugar, respetando sus elementos característicos como el techo a dos aguas y el puntal alto, así como los materiales. Todo ello en busca de una imagen contemporánea que realce los valores arquitectónicos y paisajísticos del lugar.

\section{Aumentar los servicios turístico-recreativos.}

Conjuntamente con la recuperación total del Balneario, deberá ser una prioridad la ampliación de los servicios turístico-recreativos en el territorio, especialmente los relacionados con el alojamiento y la restauración, estimulando a los trabajadores por cuenta propia para su participación en la actividad. En este sentido, sería útil aprovechar el espacio que ocupaba el antiguo Hotel Cuba, para la creación de infraestructura hotelera que garantice el hospedaje de los visitantes.

La necesidad de comunicación y acceso a Internet, es otra cuestión a tener en cuenta, por lo que se debe analizar la importancia de disponer de espacios específicos, como centros de negocios, un ciber-corner, teatro y parque infantil.

Es imprescindible el perfeccionamiento del sistema de transporte, elevando la frecuencia de las rutas existentes e incorporando otras nuevas que permitan la conexión terrestre del pueblo con otros territorios, facilitando el desplazamiento de los potenciales clientes del Balneario y el resto de los visitantes. Sería útil ampliar también las rutas locales e incorporar puntos de alquiler de vehículos, sean bicicletas o automóviles. Además, se requiere asfaltar correctamente la Carretera de San Miguel, la cual se encuentra en estado regular.

Es provechoso también que se realizaran reparaciones en "La Piscina", único centro recreativo del territorio, así como también al "Cine Jacán", ampliando de esta forma la cartera de actividades del pueblo, de manera que se realice un uso más eficiente de estas instalaciones, lo cual puede ser coordinado con el Gobierno Local y la Casa de Cultura, para compartir y conservar sus valores patrimoniales.

Sin duda, lo más importante para llevar a cabo este proceso es la existencia de voluntad política y la involucración consciente de los principales órganos de dirección, locales y regionales, en la implementación de los programas establecidos, estimulando con su participación a otros actores locales para la transformación paulatina de la comunidad en un pueblo de interés turístico.

\section{Promocionar los valores culturales del territorio.}

Sería importante y de gran interés fomentar las actividades culturales tradicionales y locales que se realizan, ya sean de carácter literario, canto, danza u otras.

Potenciar las actividades de folclore también dará al pueblo una peculiaridad especial y, al mismo tiempo, una novedad y satisfacción al visitante, enseñándole algunos de los rituales afrocubanos que posee parte de la población. 
El hecho de que San Miguel sea el único pueblo de la provincia que atrae visitantes foráneos que peregrinan al Cristo Jacán durante la Semana Santa, evidencia su gran valor religioso de carácter católico, el cual, por su existencia y larga historia de tradición, se debe promocionar al pueblo cubano-católico.

El valor cultural y tradicional que se debe potenciar más dentro de la localidad es la artesanía local, ya que se realiza hace más de cien años con los recursos naturales de la zona, hecho que aporta un elemento más para atraer a los visitantes. Para ello se propone la creación de un mercado de artesanía local, dónde además de la comercialización del producto, se hará una demostración del oficio, y la creación de una feria para reunir a todos los artesanos y compradores de la provincia; será la mejor forma de ganar fama dentro de este ámbito.

Con este espacio se pretende resolver muchos de los problemas que actualmente afectan al poblado, que van desde la falta de espacios públicos hasta el descuido de las tradiciones locales.

\section{Proponer recorridos turísticos en la zona.}

Las rutas turísticas son la mejor manera de ensamblar los recursos del territorio, ya que son la suma de los valores simbólicos vinculados con la cultura, la tradición y el patrimonio. La creación de éstas, se consideran de alta prioridad y fácil de llevar a cabo, pues no requiere de gran capital inicial.

Para el diseño e implementación de estos recorridos, se parte del supuesto de la plena recuperación, constructiva y funcional, de todos los elementos involucrados en el proceso, así como la puesta en práctica de los lineamientos anteriormente explicados. Juega un papel especial, el Balneario de San Miguel, el cual constituye el eje central de la actividad, y los servicios de restauración. Las excursiones se proponen de forma exclusiva dentro de asentamiento urbano; no obstante, se pretende de forma paulatina se extiendan a otros lugares dentro del municipio, que también resultan atractivos al turismo y, en un futuro, la coordinación de estas actividades con el resto de los municipios de Matanzas.

De esta forma, San Miguel de los Baños se reintegrará, paulatinamente, a los destinos turísticos de nuestro país como "El Paraíso de Cuba" que fue en el pasado.

Con la aplicación de esta propuesta integral se alcanzará una mayor diversificación de la Cartera de Productos de Cuba como destino turístico, específicamente en la provincia de Matanzas, ya que sus ofertas se centran principalmente en Varadero y el Gran Parque Península de Zapata. Además, se beneficiaría no sólo la población sanmigueleña y los residentes de localidades cercanas, sino que gradualmente volvería a ser un referente para todo nuestro país, contribuyendo a la rehabilitación de personas con enfermedades osteomioarticulares y dermatológicas, mejorando la salud de gran parte de la población cubana que está envejecida, y en su mayoría presenta algunos de estos padecimientos. Posibilitaría el ingreso de divisas, perfeccionando la economía local y regional; la creación 
de nuevos puestos de trabajo, nuevas ofertas de recreación, y otras condiciones que mejorarían la calidad de vida de la comunidad y los índices de desarrollo local en el territorio.

\section{Conclusiones.}

- San Miguel de los Baños todavía conserva el estilo pintoresco que gozó durante muchas décadas y todavía se está a tiempo de salvarlo. Podría convertirse en uno de los pueblos más bellos y atractivos de toda Cuba, si existiera la voluntad y los recursos para restaurarlo.

- La evaluación de las condiciones turístico-recreativas de San Miguel de los Baños permitió realizar una propuesta para su desarrollo como pueblo de interés turístico, a través de lineamientos generales. Éstos constituyen un antecedente para la conformación de estrategias y acciones que favorezcan el rescate de la localidad.

- San Miguel de los Baños es un territorio que posee gran variedad de valores únicos que lo singularizan en el contexto de la provincia de Matanzas, destacándose sus recursos turísticos naturales (fundamentalmente sus aguas mineromedicinales), histórico-culturales y arquitectónicos, lo que evidencia un innegable potencial turístico.

- A pesar de los atributos que presenta el poblado para la realización de actividades vinculadas al turismo y la recreación, no existe un favorable aprovechamiento de sus recursos, por tanto, se hace necesario la puesta en ejecución de acciones para el desarrollo y consolidación de éstos.

- La infraestructura de servicios hoteleros y extrahoteleros es insuficiente, por lo que el pueblo debe de equiparse adecuadamente, para satisfacer la potencial demanda de clientes nacionales e internacionales.

- Los atributos que posee San Miguel, hacen de él, un pueblo con elevado grado de autenticidad, suficiente para ofertar no solo productos de salud, sino también atraer visitantes en busca de turismo cultural y de naturaleza o comunitario; tributando a la diversificación de la cartera de productos de la región.

\section{Referencias bibliográficas.}

Álvarez, R. \& Espinosa, Y. Turismo y Desarrollo Local. Proyecto para convertir Cocodrilo en Pueblo Turístico Sostenible en un Área Protegida de la Isla de la Juventud (Cuba). Cuadernos de Turismo No. 22, pp 9-33. Universidad de Murcia, España (2008).

Amara. Ingeniería de Marketing. Tendencias del turismo 2018. ¿Qué puede hacer tu hotel para aumentar reservas? Recuperado el 18 de enero de 2018, de amara: https://blog.amara-marketing.com/tendencias-turismo-2018. (2017).

Arévalo, G. J. \& Guerrero, H. R. Turismo de salud por medio del aprovechamiento de aguas termales. Caso de la Ruta de la Salud Michoacán. Recuperado el 8 de febrero de 2018, de Sistema de Información Científica Redalyc: http://www.redalyc.org/html/510/51033723007/. (2014). 
Auren. Turismo de Salud en España. Escuela de Organización Indutrial \& Ministerio de Energía, Industria y Turismo. Madrid, España. (2013).

Ayala, H. S. et al. Modalidades Turísticas. Características y situación actual. La Habana, Cuba: Félix Varela. (2007).

Azteca Noticias. ¿Qué es un pueblo mágico y cómo se elige? Recuperado el 19 de febrero de 2018, de Azteca Noticias: http://www.bbc.com/mundo/noticias/2015/10/151016_mexico_pueblos_magicos_cu ltura_turismo_an. (2015)

Bautista, A., Ramón, F. \& Santandreu, C. El turismo de salud: situación actual y retos de futuro en la Comunitat Valenciana (España). Universitat Politècnica de València, España. (2016).

Bermello, Y. A tono con los nuevos tiempos. Recuperado el 13 de enero de 2018, de Semanario Económico y Financiero de Cuba: http://www.opciones.cu/cuba/2017-0929/a-tono-con-nuevos-tiempos/. (2017).

Biosphere Responsible Tourism. 22 Beneficios del Turismo Sostenible. Recuperado el 23 de enero de 2018, de Biosphere Responsible Tourism: https://www.biospheretourism.com/es/blog/22-beneficios-del-turismo-sostenible/94. (2017).

Boisier, S. Desarrollo (local): ¿de qué estamos hablando? Editorial Homo Sapiens, Rosario, Chile. (2001).

Borja, B. La evolución del turismo sostenible. Recuperado el 24 de enero de 2018, de twenergy: https://twenergy.com/a/la-evolucion-del-turismo-sostenible-2594. (2017).

Branco, P. L., Henn, M. R., Alén, M. E., \& Gonçalves, J. M. El turismo de salud y el uso terapéutico del agua. Monografía, Universidad de Málaga, Universidad de Vigo, Universidad Federal de Paraná, España. (2011).

Casali, A. ¿Qué es el desarrollo local y para qué sirve? CEDEL: Centro de Estudios para el Desarrollo Local. Buenos Aires, Argentina. (2009).

ConocelaProvincia. Pueblos Turísticos. Recuperado el 8 de febrero de 2018, de CONOCELAPROVINCIA El Blog de Buenos Aires: http://www.conocelaprovincia.com.ar/buenos_aires/pueblos/pueblos_turisticos/. (2015). 
Denis, E. Rehabilitación de Centros Históricos en la Provincia de Matanzas. Ilustraciones para un Rescate Integral del Patrimonio. Trabajo de Diploma, ISPJAE, La Habana, Cuba. (2008).

EUTM (Escuela Universitaria de Turismo de Murcia). El turismo. Concepto y definiciones e importancia actual. Murcia, España: Universidad de Murcia. (2009).

García, A. Termalismo y Deporte. Hidrocinesiterapia. Balneoterapia.Instituto Andaluz del Deporte. Consejería de Turismo, Comercio y Deporte. España. (2006).

Hernández, D. Potencialidades Turísticas de San Miguel de los Baños (Cuba). Recuperado el 17 de octubre de 2017, de Monografías: http://www.monografias.com/trabajos105/potencialidades-turisticas-san-miguelbanos/potencialidades-turisticas-san-miguel-banos2.shtml. (2015).

Jiménez, D. Las 10 características del turismta del siglo XXI. Recuperado el 17 de octubre de 2017, de El blog de Diego Jiménez Rubio: https://diegoturimarketing.com/2015/12/25/las-10-caracteristicas-del-turista-delsigloxxi/. (2015).

Lorenzo, H. \& Morales, G. Del desarrollo turístico sostenible al desarrollo local. Su comportamiento complejo. Vol. 12. págs. 453-466. Recuperado el 5 de febrero de 2018, de: Pasos. Revista de Turismo y Patrimonio Cultural. Universidad de Ciego de Ávila. Ciego de Ávila, Cuba. (2014).

MINCETUR. Manual para la Formulación del Inventario de Recursos Turísticos a Nivel Nacional. (Fase I - Categorización). Perú (2006).

Mishmesh, D. Estudio de la Situación Actual y Potencial del Turismo de Salud en Canarias. Máster Oficial en Dirección y Planificación del Turismo. Universidad de La Laguna. España. (2016).

Organización Mundial del Turismo (OMT-UNWTO), Panorama OMT del turismo internacional 2017.

Ostelea. Tendencias, Perfiles y Motivaciones del Turismo de Salud y de Bienestar. School of Tourism \& Hospitality. Barcelona, España. (2017).

Parra, I., Altabás-Jorge, R., \& Muchuly, I. Proyectos socioculturales como alternativa para el desarrollo endógeno de dos comunidades de Puerto Padre, Cuba. Revista de Investigación Agraria y Ambienta.l Vol. 6 No. 1 pp 215-225. Universidad Nacional Abierta y a Distancia. Colombia (2015). 

abril-junio, 2021

Prieto, A. San Miguel de los Baños: propuesta como pueblo de interés turístico. Trabajo De Diploma en opción al título de Licenciado en Turismo. Universidad de la Habana. Facultad de Turismo (2018).

Solís, L. Turismo de Salud. Recuperado el 16 de octubre de 2017, de Monografías: http://www.monografias.com/trabajos41/turismo-de-salud/turismo-de-salud.shtml. (2006)

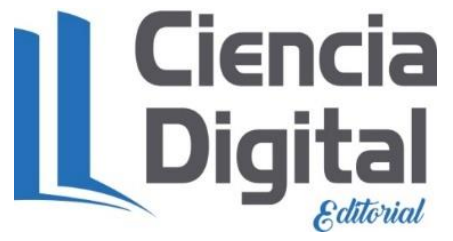




\section{Para citar el artículo indexado}

Prieto Lee, A., Martínes Carballo, M. ., \& Echarri Chávez, M. (2021). San Miguel de los Baños, un paraíso para rescatar. AlfaPublicaciones, 3(2), 77-100. https://doi.org/10.33262/ap.v3i2.45

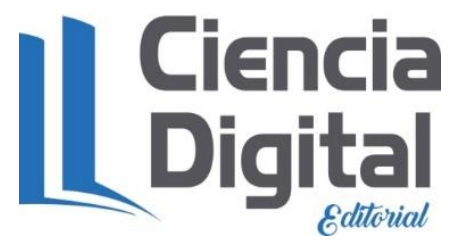

El artículo que se publica es de exclusiva responsabilidad de los autores y no necesariamente reflejan el pensamiento de la Revista Alpha Publicaciones.

El artículo queda en propiedad de la revista y, por tanto, su publicación parcial y/o total en otro medio tiene que ser autorizado por el director de la Revista Alpha Publicaciones.
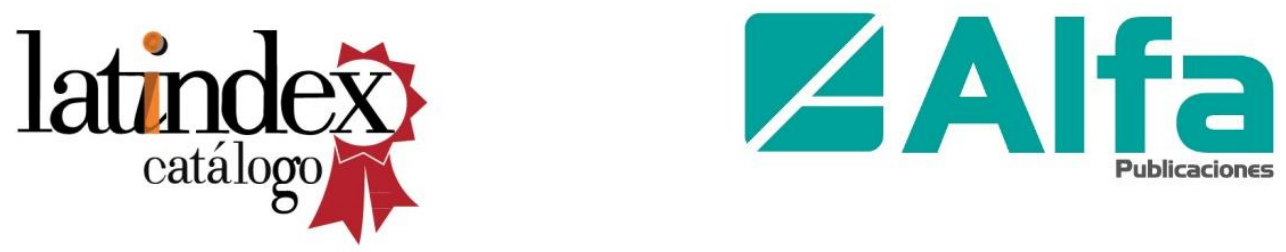